\title{
Using a New Size Dependent Orthotropic Elastic Shell Model for the Investigation of Free Vibration of Protein Microtubules
}

\author{
Yaghoub Tadi Beni \\ Faculty of Engineering, Shahrekord Universiy, Shahrekord, Iran. \\ Mehran Karimi Zeverdejani and Fahimeh Mehralian \\ Mechanical Engineering Department, Shahrekord University, Shahrekord, Iran.
}

\begin{abstract}
(Received 25 July 2016; accepted 5 October 2016)
Protein microtubules are one of the most effective intracellular components. The microtubules structure is in a manner that their behaviour is similar to that of the orthotropic materials. Therefore, in this paper, size-dependent vibration of the anisotropic protein microtubule is studied. For this purpose, using a first shear deformable shell model and based on couple stress theory, new equations are developed for the dynamic behavior of anisotropic protein microtubule. After solving the governing equations of microtubule motion, the effects of cytoplasm environment, microtubule dimensions and its mechanical properties, and material length scale parameters on the natural frequency of microtubules are investigated.
\end{abstract}

\section{INTRODUCTION}

Protein microtubules (MTs) are cylindrical bio structures which exist inside the eukaryotic cells and in the neurons of nerve cells. Microtubules are effective in the majority of intracellular interactions. Thus, to understand and prognosticate their behaviour in the face of external excitation is important. Moreover, microtubules are studied in laboratory conditions as well. MTs are the strongest intercellular elements and provide the majority of cell stiffness. Structurally, MTs are made of a peripheral connection of long fibers called protofilaments. Protofilaments, in turn, are made of a consecutive linkage of fine particles named $\alpha$ and $\beta$ tubulins. In fact, the building of MTs is similar to a cylinder to the wall of which the $\alpha$ and $\beta$ tubulins are attached. Generally, microtubules are represented by $N-S$ indices. $N$ represents the number of protofilaments, which build microtubules and the higher this index, the longer the microtubule diameter. In addition, $S$ indicates the start helix, which causes variability in tubulin arrangement in different microtubules. In fact, this index represents the angle between the protofilament axis and microtubule's central axis. Microtubules have been observed in the range of 10 nanometers to 100 microns, in terms of longitudinal dimension, and in the range of 15 to 30 nanometers, in terms of diameter. Besides, the equivalent thickness of MTs is measured as 2.7 nanometers. ${ }^{1-10}$ Considering that in the eukaryotic cells microtubules are located inside the cytoplasm environment, the surrounding cytoplasm affects the MTs' behavior. To evaluate the cytoplasm effects on the MTs behavior, the cytoplasm is modelled as a Pasternak foundation. ${ }^{11,12}$ Considering the MT dimensions, the size effect parameter must be taken into account in MT analysis. ${ }^{13-29}$ Couple stress theory is one of the higher order continuum theories capable of calculating small size effects. This theory was first presented by Mindlin and Tiersten. ${ }^{30}$ Many researchers have used couple stress theory in their studies to analyse. ${ }^{31-36}$ For instance Al-Basyouni et al. used in in order to investigate of the bending and vibration characteristic of a micro-FGM beam used couple stress scale parameters. ${ }^{33}$ So far, using different beam and cylindrical shell models, numerous studies have been conducted on the free vibration of MTs. Using a nonlocal Euler-Bernoulli beam model, Civalek, et al. investigated the free vibration of MTs. ${ }^{37}$ Using the modified strain gradient theory, Karimi and Tadi studied the effect of MT dimensions and environmental conditions on the natural frequency of MTs modelled as an EulerBernoulli beam. ${ }^{38}$ The vibration of MTs based on the nonlocal Timoshenko beam model was investigated by Heireche, et al. ${ }^{39}$ They studied the significance of the small-scale effects, shear effects, and rotary inertia on MTs vibrations. Tounsi, et al. studied the vibration and flexural rigidity of MTs using a parabolic beam model. ${ }^{40}$ They showed that the results obtained from the parabolic shear deformation beam model had a good agreement with the experimental results. Shen presented a non-linear model for the vibration analysis of MTs based on nonlocal theory. ${ }^{11}$ Tadi and Karimi analysed the vibration behavior of MTs using an isotropic shell model. ${ }^{12}$ They showed that the scale parameter in the shell model had a stronger effect on the results compared to than in the beam model. Also, by considering the shear effects and the use of couple stress theory, Baninajarian and Tadi examined the vibration characteristics and flexural rigidity of MTs. ${ }^{41}$ Reviews have demonstrated that the MTs properties in the longitudinal direction are much stronger than those in the other. ${ }^{42-44}$ Thus, in order to conduct detailed MT analysis, it is better to use anisotropic models. Wang, et al. studied the vibration of orthotropic microtubules without considering the scale parameters, while, according to the MT dimensions, the material scale parameter should have been considered. ${ }^{45}$ Recently, in order to analyse the wave propagation of MTs, Taj and Zhang presented the non-local orthotropic shell model. ${ }^{46}$ Moreover, some researchers examined MTs free vibration, based on the molecular dynamics method. Examples of those studies include the work done by Xiang and 
Liew. ${ }^{47}$ They studied MT vibration using molecular dynamics techniques. As mentioned above, MT properties vary across directions and MTs should be modelled as a size dependent anisotropic shell. According to the nature of MT structure, four characteristics affecting MT behaviour can be determined. The first characteristic is the size dependence of MTs. The second characteristic is the anisotropic behaviour of MTs. The third condition the is cylindrical shape of MTs, which cause beamlike models not to present the correct results for MTs. The fourth parameter is the shear effect in MT thickness. In fact, it can be said that due to its ignoring of shear effects, a thin shell is not a suitable model for MTs. In analytical studies, in order to consider the shear effects, there should be use of shear models, such as first shear deformation theory (FSDT). Also, in order to increase accuracy in the calculating of shear effects, researchers can make use of higher order shear deformation theory (HSDT). ${ }^{48-52}$ In recent years, a HSDT model was used by many analytical researchers. ${ }^{53-60}$ In the present work, a FSDT model is used, based on the MTs thickness to radius ratio. As mentioned in the introduction of the previous work, researchers have used different models to analyse MTs' free vibration. Initially, microtubules were modelled as macroscale cylindrical shells. ${ }^{6}$ But, these models are incapable to count the small size effects. Afterwards, researchers have used size dependent isotropic beamlike models to analyse microtubules. ${ }^{38}$ While, according to the MTs natural shape, a beam model is not appropriate for a MTs simulation. Then, in order to improve the results, nanoscale isotropic cylindrical shells were employed. ${ }^{12}$ Whereas the realistic behaviour of MTs is similar to anisotropic material. It can be said that in previous research conducted on MTs, only rarely have all the four conditions of size dependency, anisotropy, shear effects, and MT cylindrical shape been considered. In recent years, in order to obtain more realistic solutions, the nanoscale orthotropic shell was introduced, in which nanoscale effects are measured based on nonlocal theory. In the present work, attempts have been made to provide a comprehensive model of MTs. The differentiating feature of the model presented in this work is that in addition to considering the three conditions of natural MT cylindrical shape, anisotropy, and shear effects on the MT thickness, it also investigates the impact of the small-scale effects based on couple stress theory. So far, few studies have been conducted on the application of couple stress theory for anisotropic materials. Chen and Yang developed a new model to analyse a laminated composite based on couple stress theory. ${ }^{29}$ In the present work, a new model of orthotropic cylindrical shell is provided based on couple stress theory, and, by using this model, MT vibration is analysed. Thus, in this article, first, based on the energy method, and by using the calculus of variations method, the motion equations of orthotropic MTs are extracted. Afterwards, these equations are solved for MTs and in the special case of a simply-supported boundary condition, and the effects of MT mechanical properties and MT dimension ratio on the MTs natural frequency are investigated.

\section{MOTION EQUATIONS FOR SIZE DEPENDENT ANISOTROPIC MTS}

As mentioned in the previous section, MTs have a hollow cylindrical shape surrounded by cytoplasm environments in the eukaryotic cells. In physical studies, this elastic environment

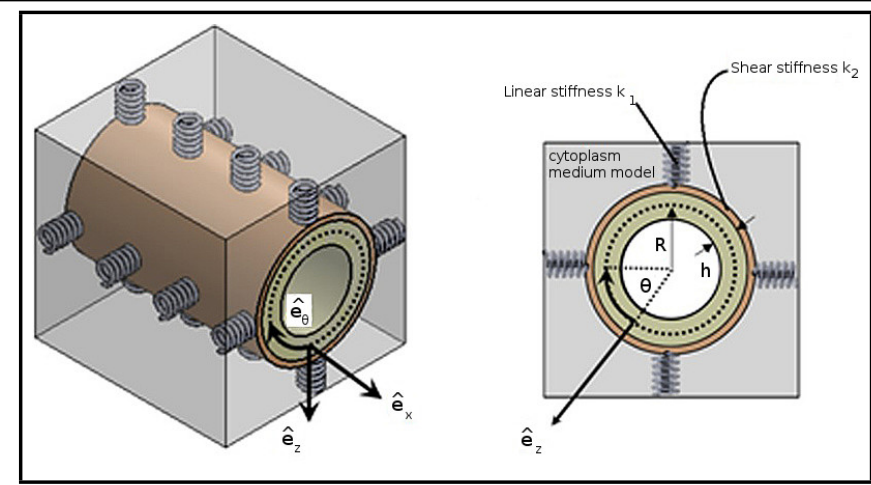

Figure 1. Protein microtubule model inside the cytoplasm.

is modelled as a Pasternak foundation, which includes linear stiffness $\left(k_{1}\right)$ and shear stiffness $\left(k_{2}\right)$ coefficients. In the cylindrical shell model, the origin of the coordinate system matches the middle cylindrical surface $(z=0)$ and the shell is configured by using the orthogonal axes $x, z$, and $\theta$. These axes represent the longitudinal, thickness and circumferential directions, respectively (Fig. 1).

Considering the ratio of MT thickness to MT radius, the FSDT model is suitable for analysis of these biostructures. In this case, the displacement field of FSDT is defined as follows:

$$
\begin{gathered}
u_{x}=u(x, \theta, t)+z \phi_{x}(x, \theta, t) ; \\
u_{\theta}=v(x, \theta, t)+z \phi_{\theta}(x, \theta, t) ; \\
u_{z}=w(x, \theta, t) .
\end{gathered}
$$

In the above equation $u, v$, and $w$ indicate the in-plane displacements. Also, $\phi_{x}$ and $\phi_{\theta}$ represent rotation around the $x$ and $\theta$ axes. Based on energy methods, Hamilton's principle is used to develop the motion equations. According to this principle, variation of the system energy in a period of time is equal to zero as follows:

$$
\delta \int_{0}^{t}\left[K-U+W_{\text {ext }}\right] d t=0 .
$$

In the above equation, $U$ expresses MTs strain energy, $K$ indicates the MTs kinetic energy, and $W_{\text {ext }}$ is the work done by external forces on the MTs. Based on couple stress theory, strain energy of MTs is obtained through following integration:

$$
U=\frac{1}{2} \int_{\Omega}[\sigma: \varepsilon+m: \chi] d V
$$

where $\varepsilon, \sigma, \chi$, and $m$ represent strain tensor, Cauchy's stress tensor, rotation gradient tensor and higher order stress tensor, respectively. Based on the equations of a general curvilinear coordinate system, the strain and rotation gradient tensors are defined as follows:

$$
\begin{gathered}
\varepsilon=\frac{1}{2}\left[\left.u^{i}\right|_{j}+\left.u^{j}\right|_{i}\right] \\
\chi=\left.\frac{1}{2}\left[\left(u_{, j}^{i}-\Gamma_{p j}^{i} u^{p}\right) \frac{e_{i j t}}{\sqrt{g}}\right]\right|_{k} .
\end{gathered}
$$

In the above equations (,) represents the ordinary derivative and $(\mid)$ denotes the covariant derivative. Also, $g_{i j}, \Gamma_{j k}^{i}$, 
and $e_{i j k}$ represent the metric tensor, second kind of Christoffel symbol, and permutation tensor, respectively. Covariant derivative for displacement vector is defined as follows:

$$
\left.u^{i}\right|_{j}=u_{, j}^{i}+\Gamma_{l j}^{i} u^{l}
$$

For a cylindrical shell, the non-zero components of the metric tensor and the second kind of Christoffel symbol, assuming $R \pm z=R$, are calculated as follows:

$$
\begin{gathered}
g_{x x}=1 ; \\
g_{z z}=1 ; \\
g_{\theta \theta}=R^{2} ; \\
\Gamma_{\theta z}^{\theta}=\frac{1}{R} ; \\
\Gamma_{\theta \theta}^{z}=-R .
\end{gathered}
$$

In the following sections, the calculation process of classic and higher-order stresses using $\varepsilon$ and $\chi$ values is addressed. For linear elastic materials, the relationship between stress and strain is expressed as follows:

$$
\sigma=C: \varepsilon
$$

In the above equation, $C$ indicates the elasticity tensor the components of which orthotropic behaviour are as follows:

$$
\begin{gathered}
C_{i j}=\left[\begin{array}{ccccc}
C_{11} & C_{12} & 0 & 0 & 0 \\
C_{21} & C_{22} & 0 & 0 & 0 \\
0 & 0 & C_{44} & 0 & 0 \\
0 & 0 & 0 & C_{55} & 0 \\
0 & 0 & 0 & 0 & C_{66}
\end{array}\right]= \\
{\left[\begin{array}{ccccc}
\frac{E_{x x}}{1-\nu_{x \theta} \nu_{\theta x}} & \frac{\nu_{x \theta} E_{\theta \theta}}{1-\nu_{x \theta} \nu_{\theta x}} & 0 & 0 & 0 \\
\frac{\nu_{\theta x} E_{x x}}{1-\nu_{x \theta} \nu_{\theta x}} & \frac{E_{\theta \theta}}{1-\nu_{x \theta} \nu_{\theta x}} & 0 & 0 & 0 \\
0 & 0 & 2 G_{\theta z} & 0 & 0 \\
0 & 0 & 0 & 2 G_{x z} & 0 \\
0 & 0 & 0 & 0 & 2 G_{x \theta}
\end{array}\right] ;}
\end{gathered}
$$

Besides, the relationship between higher-order stress and rotation gradient is defined as follows: ${ }^{61}$

$$
m=l_{i}^{2} G_{i j} \chi_{i j}+l_{j}^{2} G_{j i} \chi_{j i} .
$$

In the above equation, $l_{i}$ represents the material length scale parameter. With regard to Eq. (1) and by using Eqs. (4) to (7), components of MT strain and rotation gradient tensors are derived as follows:

$$
\begin{gathered}
\varepsilon_{x x}=u_{, x}+z \phi_{x, x} ; \\
\varepsilon_{\theta \theta}=\frac{1}{r}\left(v_{, \theta}+z \phi_{\theta, \theta}+w\right) ; \\
\varepsilon_{x z}=\frac{1}{2}\left(\phi_{x}+w_{, x}\right) ; \\
\varepsilon_{x \theta}=\frac{1}{2}\left(v_{, x}+z \phi_{\theta, x}+\frac{1}{r}\left(u_{, \theta}+z \phi_{x, \theta}\right)\right) ; \\
\varepsilon_{\theta z}=\frac{1}{2}\left(\phi_{\theta}+\frac{1}{r}\left(w_{, \theta}-v\right)\right) ; \\
\chi_{x x}=\frac{1}{2}\left[\frac{1}{r} w_{, x \theta}-\frac{1}{r} v_{, x}-\phi_{\theta, x}\right] ;
\end{gathered}
$$

$$
\begin{gathered}
\chi_{\theta \theta}=\frac{1}{2 r}\left[\phi_{x, \theta}-w_{, x \theta}+v_{, x}+z \phi_{\theta, x}-\frac{1}{r} u, \theta\right] ; \\
\chi_{z z}=\frac{1}{2}\left[\phi_{\theta, x}+\frac{1}{r^{2}} u_{, \theta}-\frac{1}{r} \phi_{x, \theta}\right] ; \\
\chi_{x \theta}=\frac{1}{2 r^{2}}\left[w_{, \theta \theta}-v_{, \theta}-r \phi_{\theta, \theta}\right] ; \\
\chi_{\theta x}=\frac{1}{2}\left[\phi_{x, x}-w_{, x x}\right] ; \\
\chi_{z x}=\frac{1}{2}\left[v_{, x x}+z \phi_{\theta, x x}-\frac{1}{r}\left(u_{, x \theta}+z \phi_{x, \theta x}\right)\right] ; \\
\chi_{z \theta}=\frac{1}{2 r}\left[v_{, x \theta}+z \phi_{\theta, \theta x}-\frac{1}{r} u_{, \theta \theta}-w_{, \theta}\right] ; \\
\left.-\frac{z}{r} \phi_{x, \theta \theta}-\phi_{x}+w_{, x}\right] .
\end{gathered}
$$

According to Eqs. (8) to (10), and by using components of strain and rotation gradient tensors, which were obtained in the Eqs. (11) and (12), components of both classical and higher order stresses are obtained as follows:

$$
\begin{aligned}
& \sigma_{x x}=\frac{E_{x x}}{1-\nu_{x} \nu_{\theta x}}\left[u_{, x}+z \phi_{x, x}\right] \\
& +\frac{\nu_{x \theta} E_{\theta \theta}}{1-\nu_{x \theta} \nu_{\theta x}}\left[\frac{1}{r}\left(v_{, \theta}+z \phi_{\theta, \theta}+w\right)\right] \text {; } \\
& \sigma_{\theta \theta}=\frac{E_{\theta \theta}}{1-\nu_{x \theta} \nu_{\theta x}}\left(\frac{1}{r}\left(v_{, \theta}+z \phi_{\theta, \theta}+w\right)\right) \\
& +\frac{\nu_{\theta x} E_{x x}}{1-\nu_{x} \nu_{\theta x}}\left(u_{, x}+z \phi_{x, x}\right) \text {; } \\
& \sigma_{x z}=\sigma_{z x}=G_{x z}\left(\phi_{x}+w_{, x}\right) \text {; } \\
& \sigma_{x \theta}=\sigma_{\theta x} \\
& =G_{x \theta}\left(v_{, x}+z \phi_{\theta, x}+\frac{1}{r}\left(u_{, \theta}+z \phi_{x, \theta}\right)\right) ; \\
& \sigma_{\theta z}=\sigma_{z \theta}=G_{\theta z}\left(\phi_{\theta}+\frac{1}{r}\left(w_{, \theta}-v\right)\right) \text {; } \\
& m_{x x}=l_{x}^{2} G_{x x}\left[\frac{1}{r} w_{, x \theta}-\frac{1}{r} v_{, x}-\phi_{\theta, x}\right] \text {; } \\
& m_{\theta \theta}=\frac{l_{\theta}^{2} G_{\theta \theta}}{r}\left[\phi_{x, \theta}-w_{, x \theta}+v_{, x}+z \phi_{\theta, x}-\frac{1}{r} u_{, \theta}\right] \text {; } \\
& m_{z z}=l_{z}^{2} G_{z z}\left[\phi_{\theta, x}+\frac{1}{r^{2}} u_{, \theta}-\frac{1}{r} \phi_{x, \theta}\right] \text {; } \\
& m_{x \theta}=m_{\theta x}=\frac{l_{x}^{2} G_{x \theta}}{2 r^{2}}\left[w_{, \theta \theta}-v_{, \theta}-r \phi_{\theta, \theta}\right] \\
& +\frac{l_{\theta}^{2} G_{\theta x}}{2}\left[\phi_{x, x}-w_{, x x}\right] ; \\
& m_{x z}=m_{z x}=\frac{l_{x}^{2} G_{x z}}{2 r^{2}}\left[v-r \phi_{\theta}-w_{, \theta}\right] \\
& +\frac{l_{z}^{2} G_{z x}}{2}\left[v_{, x x}+z \phi_{\theta, x x}-\frac{1}{r}\left(u_{, x \theta}+z \phi_{x, \theta x}\right)\right] ;
\end{aligned}
$$




$$
\begin{aligned}
m_{z \theta}=m_{\theta z}=\frac{l_{z}^{2} G_{z \theta}}{2 r}[ & v_{, x \theta}+z \phi_{\theta, \theta x}-\frac{1}{r} u_{, \theta \theta} \\
& \left.-\frac{z}{r} \phi_{x, \theta \theta}-\phi_{x}+w_{, x}\right]
\end{aligned}
$$

Finally, by substituting Eqs. (11), (12), (13), and (14) into Eq. (3), MT strain energy is obtained as in Eq. (15) (see on the next page).

After determining the MT strain energy, the kinetic energy of MTs is calculated. Using the time derivative on the FSDT displacement vector, the MT kinetic energy is computed as follows:

$$
\begin{array}{r}
K=\frac{\rho}{2} \iiint_{V}\left\{u_{, t}^{2}+z^{2} \phi_{x, t}^{2}+2 z \phi_{x, t} u_{, t}+v_{, t}^{2}\right. \\
\left.+z^{2} \phi_{\theta, t}^{2}+2 z \phi_{\theta, t} v_{, t}+w_{, t}^{2}\right\} d V .
\end{array}
$$

Now the work done by external forces on the MTs is calculated. Due to radialy displacement, the cytoplasm creates a reaction force. As mentioned in the introduction section, in order to study the cytoplasm effect on MT behavior, the cytoplasm medium is modelled as a Pasternak foundation. Researchers have demonstrated that, due to radial displacement $(w)$, a Pasternak foundation creates a reaction pressure on the body the amount of which is determined through following equation: ${ }^{11,12}$

$$
f_{\text {pas.foun }}=k_{1} w-k_{2} \nabla^{2} w
$$

Consequently, the amount of work done by external forces on the MTs can be computed as follows:

$$
W_{\text {ext }}=\frac{1}{2} \int_{V}\left[-f_{\text {pas.foun }} w R d \theta d x\right] .
$$

By substituting Eqs. (15), (16) and (18) into Eq. (1), and by using heavy simplifications, the motion equations of the new MTs model are developed as follows:

$$
\begin{array}{r}
A_{1} U_{, x x}+A_{2} U_{, x x \theta \theta}+A_{3} U_{, \theta \theta}+A_{4} U_{, \theta \theta \theta \theta} \\
+A_{5} V_{, x x x \theta}+A_{6} V_{, \theta x}+A_{7} V_{, x \theta \theta \theta}+A_{8} W_{, x} \\
+A_{9} W_{, x \theta \theta}+A_{10} \phi_{\theta, x \theta}+A_{11} \phi_{x, \theta \theta}+\rho h u_{, t t}=0 ; \\
B_{1} V_{, x x x x}+B_{2} V_{, x x}+B_{3} V_{, \theta \theta}+B_{4} V_{, x x \theta \theta}+B_{5} V \\
+B_{6} U_{, x x x \theta}+B_{7} U_{, x \theta \theta \theta}+B_{8} U_{, x \theta}+B_{9} W_{, x x \theta} \\
+B_{10} W_{, \theta \theta \theta}+B_{11} W_{, \theta}+B_{12} \phi_{\theta, \theta \theta} \\
+B_{13} \psi_{x, x \theta}+B_{14} \phi_{\theta, x x}+B_{15} \phi_{\theta}+\rho h V_{, t t}=0 ; \\
F_{1} W_{, x x x x}+F_{2} W_{, x x}+F_{3} W_{, x x \theta \theta}+F_{4} W_{, \theta \theta \theta \theta} \\
+F_{5} W_{, \theta \theta}+F_{6} W_{+}+F_{7} U_{, x}+F_{8} V_{, \theta \theta \theta}+F_{9} \psi_{\theta, \theta \theta \theta} \\
+F_{10} U_{, x \theta \theta}+F_{11} V_{, x x \theta}+F_{12} V_{, \theta}+F_{13} \phi_{x, x x x} \\
+F_{14} \phi_{x, x}+F_{15} \phi_{x, x \theta \theta}+F_{16} \phi_{\theta, \theta}+F_{17} \phi_{\theta, x x \theta} \\
+K_{1} W_{-} K_{2} W_{, x x}-\frac{1}{R^{2}} K_{2} W_{, \theta \theta}+\rho h W_{, t t}=0 ; \\
D_{1} \phi_{x, x x}+D_{2} \phi_{x, x x \theta \theta}+D_{3} \phi_{x, \theta \theta \theta \theta}+D_{4} W_{, x x x} \\
+D_{5} \phi_{x, \theta \theta}+D_{6} \phi_{x}+D_{7} \phi_{\theta, x \theta}+D_{8} \phi_{\theta, x \theta \theta \theta} \\
+D_{9} \phi_{\theta, x x x \theta}+D_{10} U_{, \theta \theta}+D_{11} W_{, x}+D_{12} W_{, x \theta \theta} \\
+D_{13} V_{, x \theta}+\rho h^{3} \phi_{x, t t}=0 ;
\end{array}
$$

$$
\begin{array}{r}
E_{1} \phi_{\theta, x x x x}+E_{2} \phi_{\theta, x x}+E_{3} \phi_{\theta, x x \theta \theta}+E_{4} \phi_{\theta, \theta \theta} \\
+E_{5} \phi_{x, x \theta \theta \theta}+E_{6} \phi_{x, x x x \theta}+E_{7} \phi_{x, x \theta}+E_{8} U_{, x \theta} \\
+E_{9} V+E_{10} W_{, \theta \theta \theta}+E_{11} V_{, x x}+E_{12} W_{, x x \theta} \\
+E_{13} W_{, \theta}+E_{14} V_{, \theta \theta}+E_{15} \phi_{\theta}+\rho h^{3} \phi_{\theta, t t}=0 .
\end{array}
$$

Coefficients ${ }_{A i j}, B_{i j}, F_{i j}, D_{i j}$, and $E_{i j}$ that can be seen in the above equations are a function of the material scale parameter and MT mechanical properties and are provided in Appendix A.

Furthermore, during the calculus of variations, the following boundary conditions have been made on the MTs boundaries.

$$
\begin{gathered}
\left.\delta u\right|_{0} ^{l}=0 \text { or }\left.\left[a_{1}+\frac{b_{7, \theta}}{2 R}\right]\right|_{0} ^{l}=0 ; \\
{\left.\left[a_{5}-\frac{b_{1}}{2 R}+\frac{b_{2}}{2 R}-\frac{b_{7, x}}{2}-\frac{b_{9, \theta}}{2 R}\right]\right|_{0} ^{l}=0 ;} \\
{\left.\left[a_{4}-\frac{b_{1, \theta}}{2 R}+\frac{b_{2, \theta}}{2 R}+\frac{b_{5, x}}{2}+\frac{b_{9}}{2 R}\right]\right|_{0} ^{l}=0 ;} \\
\left.\delta \phi_{x}\right|_{0} ^{l}=0 \text { or }\left.\left[z a_{1}+\frac{b_{5}}{2}+\frac{z b_{7, \theta}}{2 R}\right]\right|_{0} ^{l}=0 ; \\
\left.\delta \phi_{\theta, x}\right|_{0} ^{l}=0 \text { or }\left.\left[\frac{z b_{7}}{2}\right]\right|_{0} ^{l}=0 . \\
\left.\delta a_{5}-\frac{b_{1}}{2}+\frac{z b_{2}}{2 R}+\frac{b_{3}}{2}-\frac{z b_{9, \theta}}{2 R}-\frac{z b_{7, x}}{2}\right]\left.\right|_{0} ^{l}=0 ; \\
\text { or }\left.\left[\frac{b_{7}}{2}\right]\right|_{0} ^{l}=0 ; \\
\text { or }\left.\left[\frac{b_{5}}{2}\right]\right|_{0} ^{l}=0 ;
\end{gathered}
$$

Coefficients $a_{i}$ and $b_{i}$ are presented in Appendix B.

\section{SOLVING METHOD}

Based on the Navier solution method and according to FSDT cylindrical shell model, for simply supported ends, the MT displacement field solutions are considered as follows: ${ }^{32,62}$

$$
\begin{aligned}
u & =\bar{U} \cos \left(\frac{m \pi}{L} x\right) \cos (n \theta) e^{i \omega t} \\
v & =\bar{V} \sin \left(\frac{m \pi}{L} x\right) \sin (n \theta) e^{i \omega t} \\
w & =\bar{W} \sin \left(\frac{m \pi}{L} x\right) \cos (n \theta) e^{i \omega t} ; \\
\phi_{x} & =\overline{\Phi_{x}} \cos \left(\frac{m \pi}{L} x\right) \cos (n \theta) e^{i \omega t} \\
\phi_{\theta} & =\overline{\Phi_{\theta}} \sin \left(\frac{m \pi}{L} x\right) \sin (n \theta) e^{i \omega t}
\end{aligned}
$$




$$
\begin{aligned}
& U=\int_{V}\left\{\frac{E_{x x}\left(u_{, x}+z \phi_{x, x}\right)^{2}}{2\left(1-\nu_{x \theta} \nu_{\theta x}\right)}+\frac{E_{\theta \theta}\left(v_{, \theta}+z \phi_{\theta, \theta}+w\right)^{2}}{2 r^{2}\left(1-\nu_{x \theta} \nu_{\theta x}\right)}\right. \\
& +\frac{1}{2 r}\left[\frac{\nu_{x \theta} E_{\theta \theta}}{1-\nu_{x \theta} \nu_{\theta x}}+\frac{\nu_{\theta x} E_{x x}}{1-\nu_{x \theta} \nu_{\theta x}}\right]\left(u_{, x}+z \phi_{x, x}\right)\left(v_{, \theta}+z \phi_{\theta, \theta}+w\right)+\frac{1}{4} G_{\theta z}\left(\phi_{\theta}+\frac{1}{r}\left(w_{, \theta}-v\right)\right)^{2} \\
& +\frac{1}{4} G_{x \theta}\left(v_{, x}+z \phi_{\theta, x}+\frac{1}{r}\left(u_{, \theta}+z \phi_{x, \theta}\right)\right)^{2}+\frac{1}{4} G_{x z}\left(\phi_{x}+w_{, x}\right)^{2}+\frac{1}{4} l_{x}^{2} G_{x x}\left[\frac{1}{r} w_{, x \theta}-\frac{1}{r} v_{, x}-\phi_{\theta, x}\right]^{2} \\
& +\frac{1}{4 r^{2}} l_{\theta}^{2} G_{\theta \theta}\left[\phi_{x, \theta}-w_{, x \theta}+v_{, x}+z \phi_{\theta, x}-\frac{1}{r} u_{, \theta}\right]^{2}+\frac{1}{4} l_{z}^{2} G_{z z}\left[\phi_{\theta, x}+\frac{1}{r^{2}} u_{, \theta}-\frac{1}{r} \phi_{x, \theta}\right]^{2} \\
& +\frac{1}{2} l_{x}^{2} G_{x \theta}\left(\left[\frac{1}{2 r^{2}}\left(w_{, \theta \theta}-v_{, \theta}-r \phi_{\theta, \theta}\right)\right]^{2}+\left[\frac{1}{4 r^{2}}\left(w_{, \theta \theta}-v_{, \theta}-r \phi_{\theta, \theta}\right)\left(\phi_{x, x}-w_{, x x}\right)\right]\right) \\
& +\frac{1}{2} l_{\theta}^{2} G_{\theta x}\left(\left[\frac{1}{2}\left(\phi_{x, x}-w_{, x x}\right)\right]^{2}+\left[\frac{1}{4 r^{2}}\left(w_{, \theta \theta}-v_{, \theta}-r \phi_{\theta, \theta}\right)\left(\phi_{x, x}-w_{, x x}\right)\right]\right) \\
& +\frac{1}{2} l_{x}^{2} G_{x z}\left(\left[\frac{1}{2 r^{2}}\left(v-r \phi_{\theta}-w_{, \theta}\right)\right]^{2}+\left[\frac{1}{4 r^{2}}\left(v-r \phi_{\theta}-w_{, \theta}\right)\left(v_{, x x}+z \phi_{\theta, x x}-\frac{1}{r}\left(u_{, x \theta}+z \phi_{x, \theta x}\right)\right)\right]\right) \\
& +\frac{1}{2} l_{z}^{2} G_{z x}\left(\left[\frac{1}{2}\left(v_{, x x}+z \phi_{\theta, x x}-\frac{1}{r}\left(u_{, x \theta}+z \phi_{x, \theta x}\right)\right)\right]^{2}\right. \\
& \left.+\left[\frac{1}{4 r^{2}}\left(v-r \phi_{\theta}-w_{, \theta}\right)\left(v_{, x x}+z \phi_{\theta, x x}-\frac{1}{r}\left(u_{, x \theta}+z \phi_{x, \theta x}\right)\right)\right]\right) \\
& +\frac{1}{2} l_{z}^{2} G_{z \theta}\left(\left[\frac{1}{2 r}\left(v_{, x \theta}+z \phi_{\theta, \theta x}-\frac{1}{r} u_{, \theta \theta}-\frac{z}{r} \phi_{x, \theta \theta}-\phi_{x}+w_{, x}\right)\right]^{2}\right. \\
& \left.+\left[\frac{1}{8 r^{2}}\left(v_{, x \theta}+z \phi_{\theta, \theta x}-\frac{1}{r} u_{, \theta \theta}-\frac{z}{r} \phi_{x, \theta \theta}-\phi_{x}+w_{, x}\right)\left(v_{, r \theta}+\frac{1}{r}\left(v_{, \theta}-u_{, \theta \theta}\right)\right)\right]\right) \\
& \left.+\frac{1}{2} l_{z}^{2} G_{z \theta}\left[\frac{1}{2 r}\left(v_{, x \theta}+z \phi_{\theta, \theta x}-\frac{1}{r} u_{, \theta \theta}-\frac{z}{r} \phi_{x, \theta \theta}-\phi_{x}+w_{, x}\right)\right]^{2}\right\} d V .
\end{aligned}
$$

In the above equation, $\omega$ is the MT vibration frequency. Also, $\bar{U}, \bar{V}, \bar{W}, \overline{\Phi_{x}}$, and $\overline{\Phi_{\theta}}$ represent the vibration amplitude for each of the variables. Moreover, $m$ and $n$ represent the longitudinal mode number and circumferential mode number, respectively. It should be mentioned that if the relationships expressed in Eq. (25) substitute into the boundary conditions created on the MTs boundaries, the boundary conditions will be satisfied. This confirms the accuracy of solutions considered in Eq. (25). By substituting Eq. (25) into the motion equations, the motion equations are converted to following matrix form:

$$
[S]\{A\}+[M]\{\ddot{A}\}=0 .
$$

In the above equation, $S$ and $M$ represent MT stiffness and MT mass matrices, respectively. Also, $A$ and $\ddot{A}$ represent the MT displacement vector and MT acceleration respectively. Using a derivative operation, Eq. (26) can be rewritten as follows:

$$
\left[S+I M \omega^{2}\right]\{A\}=0
$$

According to the above equation, it is clear that the vector $A=0$ satisfies Eq. (27). This solution, of course, is the trivial solution. For a comprehensive analysis of this matrix equation, however, it is necessary to seek nontrivial solutions. According to Eq. (27), it is clear that to extract non-zero solutions the following conditions must be established:

$$
\operatorname{det}\left[S+I M \omega^{2}\right]=0 .
$$

After solving Eq. (28), the MT natural frequency is obtained, which will be discussed in detail in the next section.

\section{RESULTS AND DISCUSSION}

In this section, the vibration of a simply supported microtubule embedded in surrounding elastic medium is investigated using the orthotropic shell model. In order to illustrate the validity of the current study, the results obtained by the present model are compared with those available in the literature. Afterwards, various numerical examples are proposed to investigate the influence of geometrical and material properties on the vibration behaviour of microtubules. The material and geometrical properties of a microtubule are given in Table 1. Besides, the material length scale parameter is assumed as $l_{x}=l_{\theta}=l_{z}=l$ and considered only theoretically in the interval of 0 to $5 h$ in the numerical examples proposed here and the radius is assumed to be $12.8 \mathrm{~nm}, E_{x}=1 \mathrm{GPa}$, $\alpha=E_{\theta} / E_{x}=\nu_{\theta} / \nu_{x}=0.001$, and $\beta=G_{x \theta} / E_{x}$ varying between 0.000001 and 0.001 , and shear correction factor, $k_{s}$, considered 5/6. Moreover, $m \pi R / L$ is considered as a variable between 0.001 and 0.1 since the MTs usually have a length ranging from a few microns to a few tens of microns.

\subsection{Comparison Studies}

In order to verify the correctness of the current model, since no study to date has been carried out on the vibration of MTs 
Table 1. The values of orthotropic elastic constants for MTs. ${ }^{42-45}$

\begin{tabular}{||c|c|}
\hline Parameters & Values \\
\hline Longitudinal modulus $E_{x}$ & $0.5-2 \mathrm{GPa}$ \\
\hline Circumferential modulus $E_{\theta}$ & $1-4 \mathrm{MPa}$ \\
\hline Shear modulus of microtubules $G_{x} \theta$ & $1 \mathrm{KPa}-1 \mathrm{MPa}$ \\
\hline Poisson's ratio in axial direction $\nu_{x}$ & 0.3 \\
\hline Mass density per unit volume $\rho$ & $1.47 \mathrm{~g} / \mathrm{cm}^{3}$ \\
\hline Equivalent thickness $h$ & $2.7 \mathrm{~nm}$ \\
\hline Effective thickness for bending $h_{0}$ & $1.6 \mathrm{~nm}$ \\
\hline
\end{tabular}

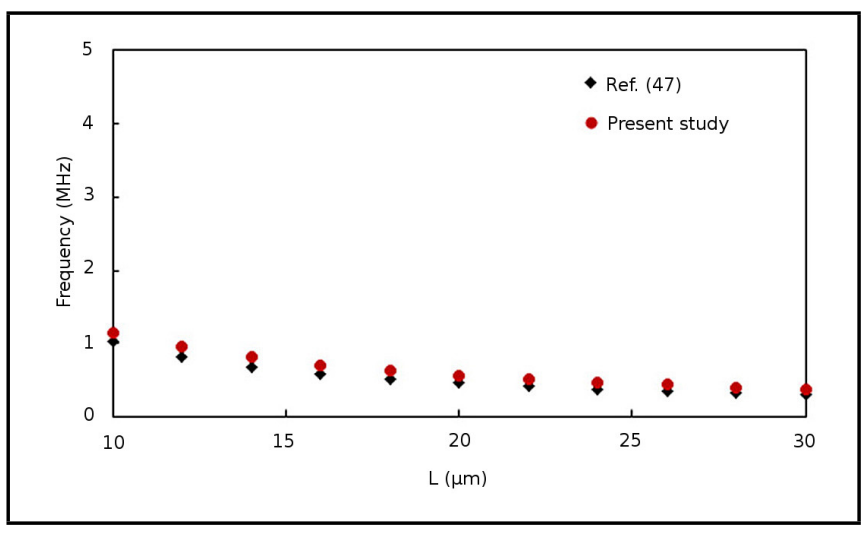

Figure 2. Comparison of the frequency of the simply supported isotropic shell.

on the basis of the new modified couple stress theory using the orthotropic shell model, the frequency response of isotropic MT is shown based on the classical continuum theory, which the equilibrium equations and boundary conditions are derived by setting the material length scale parameter to zero $(l=0)$. Therefore, the frequency of the MTs are shown in Fig. 2 and a good consistency between the obtained results and those of Xian and Liew is illustrated in this comparison. ${ }^{47}$ Since the flexural rigidity ratio as a mechanical property of MTs can be obtained through the free vibration of MTs, using the present model, the flexural rigidity ratio is compared with that of $\mathrm{Gu}$, et al. ${ }^{9}$ and Pampaloni, et al. ${ }^{63}$ in Fig. 3. As it is visible, a good consistency between the obtained results and those of $\mathrm{Gu}$, et al. ${ }^{9}$ and Pampaloni, et al. ${ }^{63}$ is illustrated in this comparison; besides, the results corresponding to $l=3 h$ are the most accurate ones compared to the experimental data. Therefore, the current model has supremacy rather than the previous FSDT shell and Timoshenko beam models, as it can fill the gaps between the analytical and experimental data at the nano scale of MTs.

\subsection{Effects of the Shear Modulus on the Fre- quency}

Figure 4 shows the natural frequencies of an isolated MT given by orthotropic shell model for variable shear modulus ratio. As it can be seen, the frequency increases by increasing the shear modulus ratio, with regard to the changing of the $(m \pi R / L)$ of the MT. Moreover, the increase of the frequencies is intensified by the increase in $(m \pi R / L)$. On the other hand, if $\beta=0.0001$ and $(m \pi R / L)$ is less than 0.01 , the frequencies predicted by $\beta=0.0001$ and $\beta=0.001$ are very close to each other. This concludes that, if $\beta=0.0001$ frequencies predicted by the orthotropic shell model are similar to that of $\beta=0.001$ for sufficiently long MTs $((m \pi R / L) ; 0.01$, or $L>4 m \mu \mathrm{m})$.

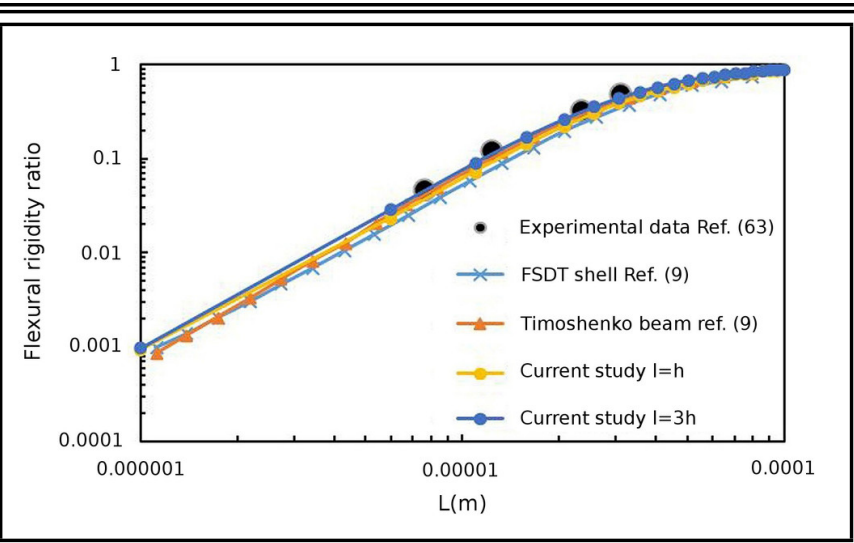

Figure 3. Comparison between the flexural rigidity ratio- length relations of an isolated microtubule given by FSDT based on NMCST in current study and FSDT shell model, Timoshenko beam model and experimental data.

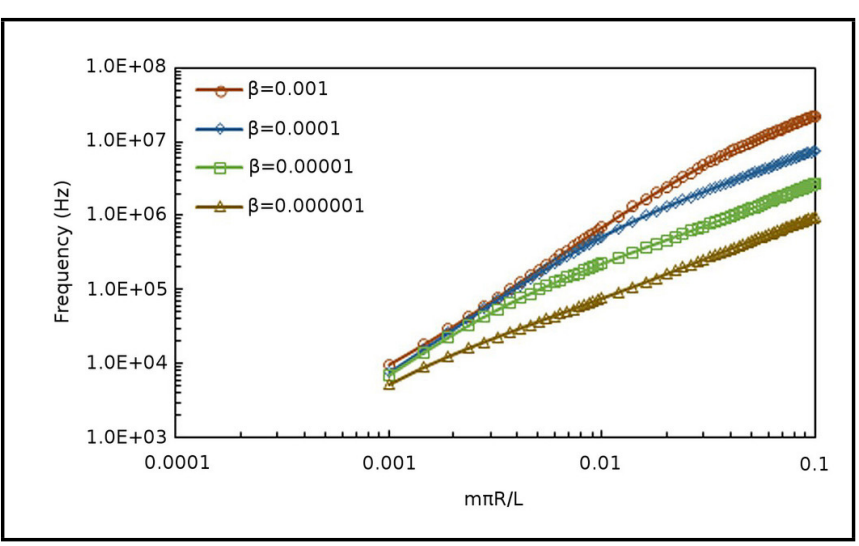

Figure 4. Dispersion relation of an isolated MT given by orthotropic shell model for different shear modulus ratio.

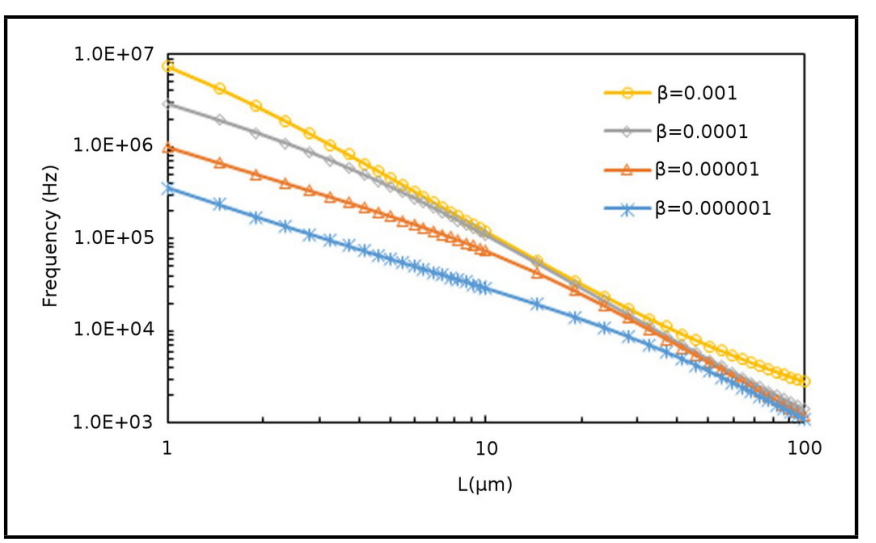

Figure 5. Length dependence of frequency of an isolated MT, given by orthotropic shell model.

\subsection{Effects of Length on Frequency}

To illustrate the influence of length on the frequencies of MT, Figs. 5 and 6 plot the frequency with respect to length for different shear modulus ratios and length scale parameters, respectively. As is visible, the increase in length leads to a decrease in MT stiffness and consequently, reduces the frequency; in addition, the decrease in frequency is intensified by an increase in shear modulus and material length scale parameters. Such that, in $l=5 h$, the reduced rate of frequency is 0.93 ; while in $l=0$ this rate is about 0.079 . 


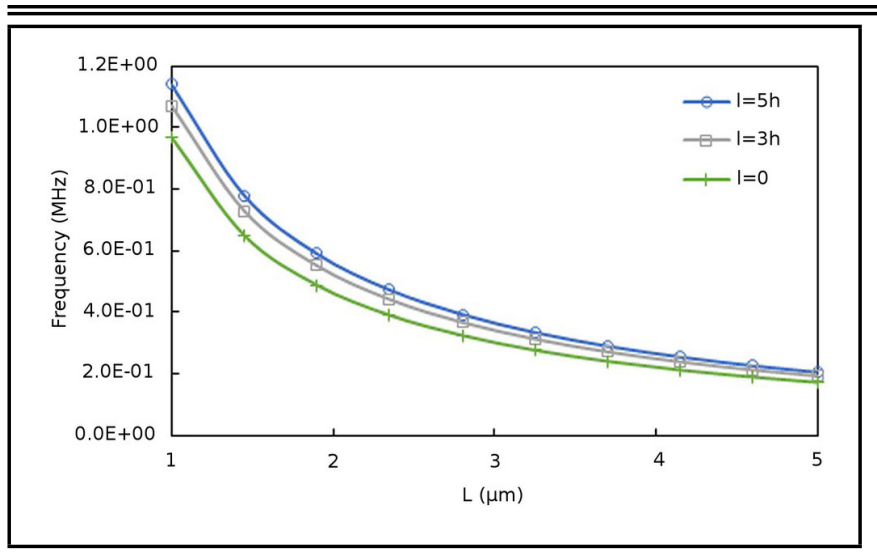

Figure 6. Frequency for an isolated MT given by orthotropic shell model in different length scale parameters.

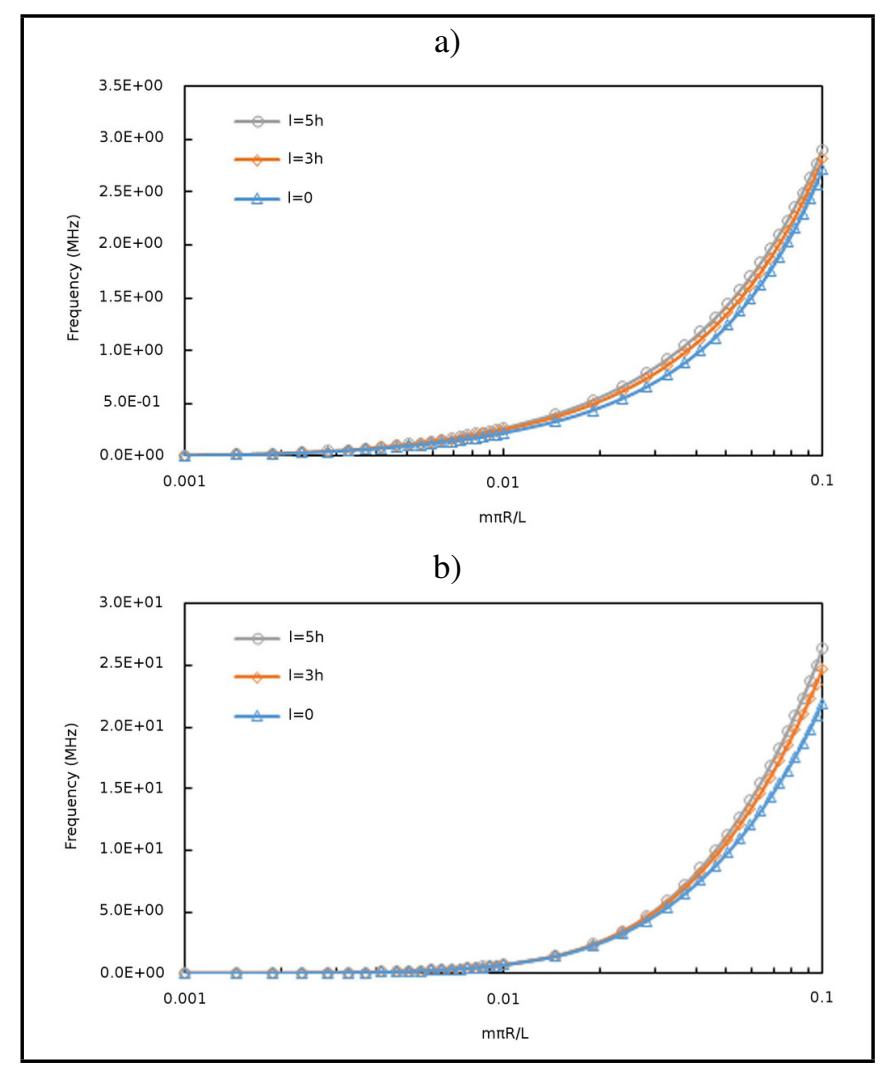

Figure 7. The dispersion curves of the microtubule frequencies for different length scale parameter, a) $\beta=0.00001$, b) $\beta=0.001$.

\subsection{Effects of the Material Length Scale Parameter on the Frequency}

Figures $7(a-b)$ illustrate the influence of material length scale parameter on the frequency of MT through $(m \pi R / L)$ distribution for $\beta=0.00001$ and $\beta=0.001$, respectively. As it can be seen, the frequency is size dependent; such that, an increase in the material length scale parameter increases the frequency. Besides, according to both illustrations, the increasing effect of the material length scale parameter on the frequency is intensified by an increase in $(m \pi R / L)$. So that, the size effect is more significant for $L<4 m$ or $(m \pi R / L)>0.01$ in $\beta=0.00001$ and for $L<1.2 m$ or $(m \pi R / L)>0.0325$ in $\beta=0.001$, according to Figs. 6(a-b).

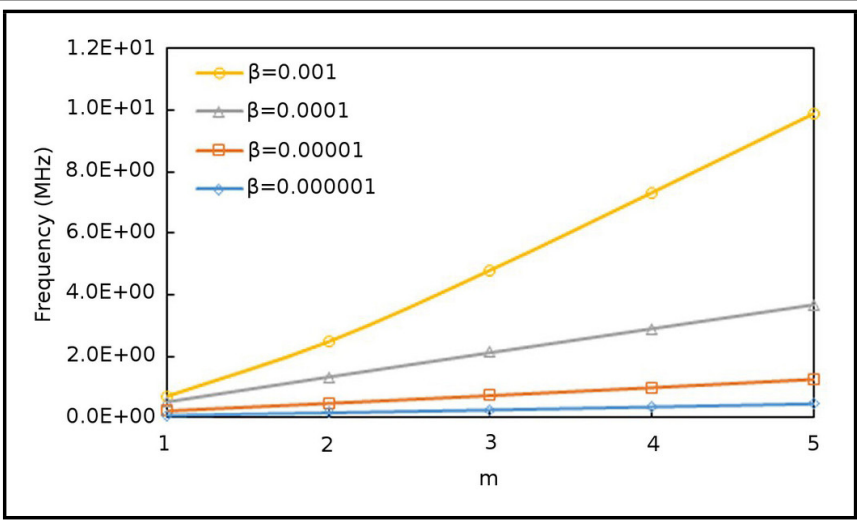

Figure 8. Variation in frequency with vibrational mode numbers (m) for MTs for different shear modulus ratio.

Table 2. Vibration frequency (MHz) for different environmental condition.

\begin{tabular}{||c|c|c|c||c|c|c||}
\hline \multirow{3}{*}{$\beta$} & \multicolumn{3}{|c|}{$\left(k_{w}, k_{g}\right)=(0,0)$} & \multicolumn{3}{c|}{$\left(k_{w}, k_{g}\right)=(2.7,0.27)$} \\
\cline { 2 - 7 } & \multicolumn{3}{|c|}{$l / h$} & \multicolumn{3}{c|}{$l / h$} \\
\cline { 2 - 7 } & 0 & 3 & 5 & 0 & 3 & 5 \\
\hline 0.000001 & 0.9598 & 0.9616 & 0.9641 & 90.935 & 90.936 & 90.937 \\
\hline 0.00001 & 2.7131 & 2.8125 & 2.9032 & 90.972 & 90.983 & 90.996 \\
\hline 0.0001 & 7.4701 & 8.3533 & 8.9168 & 91.256 & 91.403 & 91.548 \\
\hline 0.0001 & 21.943 & 24.752 & 26.388 & 93.731 & 94.894 & 95.682 \\
\hline
\end{tabular}

\subsection{Effects of the Mode Number on the Frequency}

The frequency versus mode numbers of MT is plotted for different shear modulus ratio in Fig. 8. Clearly, the frequency value increases when the mode number increases; moreover, by an increase in the shear modulus ratio, the influence of the mode number on the frequency becomes more and more prominent. So that, when $\beta=0.001$, the frequency increases from 0.69 to $9.88 \mathrm{MHz}$; however, if $\beta=0.000001$ the frequency changes from 0.076 to $0.45 \mathrm{MHz}$.

\subsection{Effects of the Cytoplasm Medium on the Frequency}

As mentioned previously, the Pasternak foundation is utilized to investigate the effect of the elastic medium surrounding MT. Table 2 shows the frequency of the MT being outside cytoplasm more $\left(k_{w}, k_{g}\right)=(0,0)$ and inside Pasternak cytoplasm $\left(k_{w}, k_{g}\right)=(2.7 \mathrm{KPa} / \mathrm{nm}, 0.27 \mathrm{KPa} \cdot \mathrm{nm})$. The results are also listed for MT in Table 2. It is found that the elastic medium surrounding the MT increases the MT stiffness and consequently increases the frequency of the MT inside the cytoplasm more than the MT outside the cytoplasm. Through Table 3, the influence of the length scale parameter on the frequency of MTs, with respect to foundation parameters and mode numbers is illustrated in detail. This table has a trend similar to Fig. 6, as such an increase in the length scale parameter increases frequency of MTs and as it is visible, increasing the effect of the length scale parameter on the frequency is intensified in higher mode numbers, in spite of the foundation parameter, which doesn't increase the influence of the length scale parameter on the frequency of MTs significantly. 
Table 3. Vibration frequency (MHz) for different environmental condition in higher mode numbers.

\begin{tabular}{|c|c|c|c||c|c|c||}
\hline \multirow{3}{*}{$l / h$} & \multicolumn{3}{|c|}{$\left(k_{w}, k_{g}\right)=(0,0)$} & \multicolumn{3}{|c|}{$\left(k_{w}, k_{g}\right)=(2.7,0.27)$} \\
\cline { 2 - 7 } & \multicolumn{3}{|c|}{$(m, n)$} & \multicolumn{3}{|c|}{$(m, n)$} \\
\cline { 2 - 7 } & $(1,1)$ & $(2,2)$ & $(3,3)$ & $(1,1)$ & $(2,2)$ & $(3,3)$ \\
\hline 0 & 2.7131 & 20.740 & 50.340 & 90.972 & 118.71 & 134.04 \\
\hline 1 & 2.7274 & 25.974 & 63.737 & 90.973 & 119.73 & 139.71 \\
\hline 2 & 2.7646 & 36.335 & 88.306 & 90.977 & 122.37 & 152.59 \\
\hline 3 & 2.8125 & 46.822 & 110.83 & 90.983 & 125.82 & 166.60 \\
\hline
\end{tabular}

\section{CONCLUSIONS}

In this paper, the free vibration of protein microtubule inside the cytoplasm was examined. For this purpose, the MT is modelled as FSDT. Also, the couple stress theory was employed to measure the MT small-scale effect. First, in order to evaluate the results in a more efficient fashion, the results were compared to those of other studies and experiments, and their accuracy and precision were confirmed. Besides, the current model has supremacy over the previous FSDT shell and Timoshenko beam models, as it can fill the gaps between the analytical and experimental data at the nano scale of MTs. After solving the MT free vibration equations, the results demonstrated that an increase in the modulus ratio and MT length leads to an increase and decrease in MT frequency, respectively. Furthermore, it was determined that although the scale parameter invariably increases MT frequency, the intensity of the effect of this parameter for MTs with $(m \pi R / L)>0.01$, the condition is stronger. In addition, it is demonstrated that the impact of increased mode number on MT frequency is stronger for higher modulus ratios. Also, the impact of the cytoplasm medium on MT frequency was investigated and the results indicated that cytoplasm strongly increases MT vibration frequency by increasing MT stiffness. Also, it was found that the impact of the length scale parameter on the MTs frequency is stronger for a higher vibration mode number.

\section{ACKNOWLEDGEMENTS}

This work has been financially supported by the research deputy of Shahrekord University. The grant number was 94GRD1M835.

\section{REFERENCES}

1 Hawkins, T., Mirigian, M., Yasar, M. S., and Ross, J. L. Mechanics of microtubules, Journal of Biomechanics, 43 (1), 23-30, (2010), https://dx.doi.org/10.1016/j.jbiomech.2009.09.005.

2 Kis, A., Kasas, S., Babic, B., Kulik, A. J., Benolt, W., Briggs, G. A, Schonenberger, C., Catsicas, S., and Forro, L. Nanomechanics of microtubules, Physical Review Letters, 89, 248101, (2002), https://dx.doi.org/10.1103/PhysRevLett.89.248101.

3 Xiang, P. and Liew, K. M. Dynamic behaviours of long and curved microtubules based on an atomistic-continuum model, Comput. Methods Appl. Mech. Eng, 223-224, 123132, (2012), https://dx.doi.org/10.1016/j.cma.2012.02.023.

4 Alberts, B., Bray, D., Lewis, J., Raff, M., Roberts, K., and Watson, J. Molecular biology of the cell,
Third ed. Garland Publishing, New York, (1994), https://dx.doi.org/10.1002/bmb.2003.494031049999.

5 Chretien, D. and Fuller, D. S. Microtubules switch occasionally into unfavourable configurations during elongation, Journal of Molecular Biology, 298 (4), 663-676, (2000), https://dx.doi.org/10.1006/jmbi.2000.3696.

${ }^{6}$ Huang, G. Y., Mai, Y. W., and Ru, C. Q. Surface deflection of a microtubule loaded by a concentrated radial force, Nanotechnology, 19, 125101, (2008), https://dx.doi.org/10.1088/0957-4484/19/12/125101.

7 Gao, Y. and An, L. A nonlocal elastic anisotropic shell model for microtubule buckling behaviours in cytoplasm, Physica E: Low-dimensional Systems and Nanostructures, 42 (9), 2406-2415, (2010), https://dx.doi.org/10.1016/j.physe.2010.05.022.

8 Shen, H. S. Nonlocal shear deformable shell model for bending buckling of microtubules embedded in an elastic medium, Physics Letter A, 374 (39), 4030-4039, (2010), https://dx.doi.org/10.1016/j.physleta.2010.08.006.

9 Gu, B., Mai, Y. W., and Ru, C. Q. Mechanics of microtubules modelled as orthotropic elastic shells with transverse shearing, Acta Mech., 207 (3-4), 195-209, (2009), https://dx.doi.org/10.1007/s00707-008-0121-8.

10 Akgoz, B. and Civalek, O. A new trigonometric beam model for buckling of strain gradient microbeams, International Journal of Mechanical Sciences, 81, 88-94, (2014), https://dx.doi.org/10.1016/j.ijmecsci.2014.02.013.

11 Shen, H. S. Nonlinear vibration of microtubules in living cells, Current Applied Physics, 11 (3), 812-821, (2011), https://dx.doi.org/10.1016/j.cap.2010.11.116.

12 Tadi Beni, Y. and Karimi Zeverdejani, M. Free vibration of microtubules as elastic shell model based on modified couple stress theory, Journal of Mechanics in Medicine and Biology, 15 (3), 1550037, (2015), https://dx.doi.org/10.1142/S0219519415500372.

13 Gao, Y. and Lei, F. M. Small-scale effects on the mechanical behaviours of protein microtubules based on the nonlocal elasticity theory, Biochemical and Biophysical Research Communications, 387 (3), 467-471, (2009), https://dx.doi.org/10.1016/j.bbrc.2009.07.042.

14 Civalek, O., Demir, Ç. Bending analysis of microtubules using nonlocal Euler-Bernoulli beam theory, Applied Mathematical Modelling, 35 (5), 2053-2067, (2011), https://dx.doi.org/10.1016/j.apm.2010.11.004.

15 Akg:oz, B., Civalek, O. Application of strain gradient elasticity theory for buckling analysis of protein microtubules, Current Applied Physics, 11 (5), 1133-1138, (2011), https://dx.doi.org/10.1016/j.cap.2011.02.006.

16 Civalek, O. and Akgoz, B. Static analysis of single walled carbon nanotubes (SWCNT) based on Eringen's nonlocal elasticity theory, International Journal of Engineering and Applied Sciences, 1 (2), 47-56, (2009). 
17 Demir, C. and Civalek, O. Torsional and longitudinal frequency and wave response of microtubules based on the nonlocal continuum and nonlocal discrete models, Applied Mathematical Modelling, 37 (22), 9355-9367, (2013), https://dx.doi.org/10.1016/j.apm.2013.04.050.

18 Tadi Beni, Y., Abadyan, M. R., and Noghrehabadi, A. Investigation of size effect on the pull-in instability of beamtype NEMS under van der Waals Attraction, Procedia Engineering, 10, 1718-1723, (2011), https://dx.doi.org/10.1016/j.proeng.2011.04.286.

19 Mohammadi Dashtaki, P. and Tadi Beni, Y. Effects of Casimir force and thermal stresses on the buckling of electrostatic nano-bridges based on couple stress theory, Arabian Journal for Science and Engineering, 39 (7), 57535763, (2014), https://dx.doi.org/10.1007/s13369-014-11076.

20 Tadi Beni, Y., Karimipour, I., and Abadyan, M. Modelling the effect of intermolecular modelling force on the size-dependent pull-in behaviour of beam-type NEMS using modified couple stress theory, Journal of Mechanical Science and Technology, 28 (9), 3749-3757, (2014), https://dx.doi.org/10.1007/s12206-014-0836-5.

21 Zeighampour, H. and Tadi Beni, Y. Free vibration analysis of axially functionally graded nanobeam with radius varies along the length based on strain gradient theory, Applied Mathematical Modelling, 39 (18), 5354-5369, (2015), https://dx.doi.org/10.1016/j.apm.2015.01.015.

22 Tadi Beni, Y. Size-dependent electromechanical bending, buckling, and free vibration analysis of functionally graded piezoelectric nanobeams, Journal of Intelligent Material Systems and Structures, 27 (16), (2016), https://dx.doi.org/10.1177/1045389X15624798.

23 Mehralian, F. and Tadi Beni, Y. Size-dependent torsional buckling analysis of functionally graded cylindrical shell, Composites Part B: Engineering, 94, 11-25, (2016), https://dx.doi.org/10.1016/j.compositesb.2016.03.048.

24 Tadi Beni, Y. A nonlinear electro-mechanical analysis of nanobeams based on the size-dependent piezoelectricity theory, Journal of mechanics, 33 (3), 289-301, (2016), https://dx.doi.org/10.1017/jmech.2016.65.

25 Tadi Beni, Y. Size-dependent analysis of piezoelectric nanobeams including electro-mechanical coupling, $\mathrm{Me}$ chanics Research Communications, 75, 67-80, (2016), https://dx.doi.org/10.1016/j.mechrescom.2016.05.011.

26 Sedighi, HM., Daneshmand, F., and Abadyan, M. Modelling the effects of material properties on the pull-in instability of nonlocal functionally graded nano-actuators, ZAMM Journal of applied mathematics and mechanics: Zeitschrift fur angewandte Mathematik und Mechanik, 96 (3), 385-400, (2016), https://dx.doi.org/10.1002/zamm.201400160.

27 Sedighi, HM., Daneshmand, F., and Abadyan, M. Modified model for instability analysis of symmetric FGM double-sided nano-bridge: Corrections due to surface layer, finite conductivity and size effect, Composite Structures, 132, 545-557, (2015), https://dx.doi.org/10.1016/j.compstruct.2015.05.076.

28 Sedighi, HM., Keivani, M., and Abadyan, M. Modified continuum model for stability analysis of asymmetric FGM double-sided NEMS: Corrections due to finite conductivity, surface energy and nonlocal effect, Composites Part B Engineering, 83, 117-133, (2015), https://dx.doi.org/10.1016/j.compositesb.2015.08.029.

29 Sedighi, HM., Daneshmand, F, and Abadyan, M. Dynamic instability analysis of electrostatic functionally graded doubly-clamped nanoactuators, Composite Structures, 124, 55-64, (2015), https://dx.doi.org/10.1016/j.compstruct.2015.01.004.

30 Mindlin, R. D. and Tiersten, H. F. Effects of couplestresses in linear elasticity, Archive for Rational Mechanics and Analysis, 11 (1), 415-448, (1962), https://dx.doi.org/10.1007/BF00253946.

31 Zeighampour, H. and Tadi Beni, Y. Analysis of conical shells in the framework of coupled stresses theory, International Journal of Engineering Science, 81, 107-122, (2014), https://dx.doi.org/10.1016/j.ijengsci.2014.04.008

32 Zeighampour, H. and Tadi Beni, Y. Size-dependent vibration of fluid-conveying double-walled carbon nanotubes using couple stress shell theory, Physica E: Lowdimensional Systems and Nanostructures, 61, 28-39, (2014), https://dx.doi.org/10.1016/j.physe.2014.03.011.

33 Al-Basyouni, K. S., Tounsi, A., and Mahmoud, S. R. Size dependent bending and vibration analysis of functionally graded micro beams based on modified couple stress theory and neutral surface position, Compos. Struct., 125, 621-630, (2015), https://dx.doi.org/10.1016/j.compstruct.2014.12.070.

34 Dehrouyeh Semnani, A. M., Dehrouyeh, M., Torabi Kafshgari, M., and Nikkhah Bahrami, M. A damped sandwich beam model based on symmetricdeviatoric couple stress theory, International Journal of Engineering Science, 92, 83-94, (2015), https://dx.doi.org/10.1016/j.ijengsci.2015.03.007.

35 Wanji, C., Li, X., and Xu, M. A modified couple stress model for bending analysis of composite laminated beams with first order shear deformation, Composite Structures, 93 (11), 2723-2732, (2011), https://dx.doi.org/10.1016/j.compstruct.2011.05.032.

36 Reddy, J. N., Romanoff, J., and Loya, J. A. Nonlinear finite element analysis of functionally graded circular plates with modified couple stress theory, European Journal of Mechanics - A/Solids, 56, 92-104, (2016), https://dx.doi.org/10.1016/j.euromechsol.2015.11.001.

37 Civalek, Ö., Demir, Ç., and Akg oz, B. Free vibration and bending analyses of cantilever microtubules based on nonlocal continuum model, Mathematical and Computational Applications, 15 (2), 289-298, (2010), https://dx.doi.org/10.3390/mca15020289. 
38 Karimi Zeverdejani, M. and Tadi Beni, Y. The nano scale vibration of protein microtubules based on modified strain gradient theory, Current Applied. Physics, 13 (8), 1566-1576, (2013), https://dx.doi.org/10.1016/j.cap.2013.05.019.

39 Heireche, H., Tounsi, A., Benhassaini, H., Benzair, A., Bendahmane, M., Missouri, M., and Mokadem, S. Nonlocal elasticity effect on vibration characteristics of protein microtubules, Physica E, 42 (9), 2375-2379, (2010), https://dx.doi.org/10.1016/j.physe.2010.05.017.

40 Tounsi, A., Heireche, H., Benhassaini, H., and Missouri, M. Vibration and length-dependent flexural rigidity of protein microtubules using higher order shear deformation theory, Journal of Theoretical Biology, 266 (2), 250-255, (2010), https://dx.doi.org/10.1016/j.jtbi.2010.06.037.

41 Baninajjaryan, A. and Tadi Beni, Y. Theoretical study of the effect of shear deformable shell model, elastic foundation and size dependency on the vibration of protein microtubule, Journal of Theoretical Biology, 382, 111-121, (2015), https://dx.doi.org/10.1016/j.jtbi.2015.06.038.

42 Shi, Y. J., Guo, W., and Ru, C. Q. Relevance of Timoshenko-beam model to microtubules of low shear modulus, Physica E: Low-dimensional Systems and Nanostructures, 41 (2), 213-219, (2008), https://dx.doi.org/10.1016/j.physe.2008.06.025.

43 Wang, C. Y., Ru, C. Q., and Mioduchowski, A. Orthotropic elastic shell model for buckling of microtubules, Physical Review E, 74, 052901, (2006), https://dx.doi.org/10.1103/PhysRevE.74.052901.

44 Qian, X. S., Zhang, J. Q., and Ru, C.Q. Wave propagation in orthotropic microtubules, J. Appl. Phys., 101, 8470284717, (2007), https://dx.doi.org/10.1063/1.2717573.

45 Wang, C. Y., Ru, C.Q., and Mioduchowski, A. Vibration of microtubules as orthotropic elastic shells, Physica E: Lowdimensional Systems and Nanostructures, 35 (1), 48-56, (2006), https://dx.doi.org/10.1016/j.physe.2006.05.008.

46 Taj, M. and Zhang, J. Analysis of wave propagation in orthotropic microtubules embedded within elastic medium by Pasternak model, Journal of the Mechanical Behavior of Biomedical Materials, 30, 300-305, (2014), https://dx.doi.org/10.1016/j.jmbbm.2013.11.011.

47 Xiang, P. and Liew, K. M. Free vibration analysis of microtubules based on an atomistic-continuum model, Journal of Sound and Vibration, 331 (1), 213-230, (2012), https://dx.doi.org/10.1016/j.jsv.2011.08.024

48 Bourada, M., Kaci, A., Houari, M. S. A., and Tounsi, A. A new simple shear and normal deformations theory for functionally graded beams, Steel and Composite Structures, 18 (2), 409-423, (2015), https://dx.doi.org/10.12989/scs.2015.18.2.409.

49 Belabed, Z., Houari, M. S. A., Tounsi, A., Mahmoud, S. R., and Beg, A. An efficient and simple higher order shear and normal deformation theory for functionally graded material
(FGM) plates, Composites: Part B, 60, 274-283, (2014), https://dx.doi.org/10.1016/j.compositesb.2013.12.057.

50 Hebali, H., Tounsi, A., Houari, M. S. A., Bessaim, A., and Adda Bedia, E. A. A new quasi-3D hyperbolic shear deformation theory for the static and free vibration analysis of functionally graded plates, ASCE J. Engineering Mechanics, 140 (2), 374-383, (2014), https://dx.doi.org/10.1061/(ASCE)EM.19437889.0000665 .

51 Bennoun, M., Houari, M.S.A., and Tounsi, A. A novel five variable refined plate theory for vibration analysis of functionally graded sandwich plates, Mechanics of Advanced Materials and Structures, 23 (4), 423-431, (2016), https://dx.doi.org/10.1080/15376494.2014.984088.

52 Mahi, A., Adda Bedia, E. A., and Tounsi, A. A new hyperbolic shear deformation theory for bending and free vibration analysis of isotropic, functionally graded, sandwich and laminated composite plates, Applied Mathematical Modelling, 39 (9), 2489-2508. (2015), https://dx.doi.org/10.1016/j.apm.2014.10.045.

53 Ait Yahia, S., Ait Atmane, H., Houari, M. S. A., and Tounsi, A. Wave propagation in functionally graded plates with porosities using various higher-order shear deformation plate theories, Struct. Eng. Mech., 53 (6), 1143-1165, (2015), https://dx.doi.org/10.12989/sem.2015.53.6.1143.

54 Meziane, A. A., Abdelaziz, H. H., and Tounsi, A. An efficient and simple refined theory for buckling and free vibration of exponentially graded sandwich plates under various boundary conditions, Journal of Sandwich Structures and Materials, 16 (3), 293-318, (2014), https://dx.doi.org/10.1177/1099636214526852.

55 Bellifa, H., Benrahou, K. H., Hadji, L., Houari, M. S. A., and Tounsi, A. Bending and free vibration analysis of functionally graded plates using a simple shear deformation theory and the concept the neutral surface position, J Braz. Soc. Mech. Sci. Eng., 38 (1), 265-275, (2016), https://dx.doi.org/10.1007/s40430-015-0354-0.

56 Tounsi, A., Houari, M. S. A., Benyoucef, S., and Adda Bedia, E. A. A refined trigonometric shear deformation theory for thermoelastic bending of functionally graded sandwich plates, Aerospace Sci. Tech., 24 (1), 209-220, (2013), https://dx.doi.org/10.1016/j.ast.2011.11.009.

57 Bouderba, B., Houari, M. S. A., and Tounsi, A. Thermomechanical bending response of FGM thick plates resting on Winkler-Pasternak elastic foundations, Steel and Composite Structures, 14 (1), 85-104, (2013), https://dx.doi.org/10.12989/scs.2013.14.1.085.

58 Zidi, M., Tounsi, A., Houari, M. S. A., and Beg, O. A. Bending analysis of FGM plates under hygrothermomechanical loading using a four variable refined plate theory, Aerospace Sci. Tech., 34, 24-34, (2014), https://dx.doi.org/10.1016/j.ast.2014.02.001. 
59 Hamidi, A., Houari, M. S. A., Mahmoud, S. R., and Tounsi, A. A sinusoidal plate theory with 5unknowns and stretching effect for thermomechanical bending of functionally graded sandwich plates, Steel and Composite Structures, 18 (1), 235-253, (2015), https://dx.doi.org/10.12989/scs.2015.18.1.235.

60 Bousahla, A. A., Houari, M. S. A., Tounsi, A., and Adda Bedia, E. A. A novel higher order shear and normal deformation theory based on neutral surface position for bending analysis of advanced composite plates, Int. J. Computat. Method., 11 (6), 1350082, (2014), https://dx.doi.org/10.1142/S0219876213500825.

61 Yang, S. and Chen, W. On hypotheses of composite laminated plates based on new modified couple stress theory, Composite Structures, 133, 46-53, (2015), https://dx.doi.org/10.1016/j.compstruct.2015.07.050.

62 Zeighampour, H. and Tadi Beni, Y. A shear deformable cylindrical shell model based on couple stress theory, Archive of Applied Mechanics, 85 (4), 539-553, (2015), https://dx.doi.org/10.1007/s00419-014-0929-8.

63 Pampaloni, F., Lattanzi, G., Jonas, A., Surrey, T., Frey, E., and Florin, E. Thermal fluctuation of grafted microtubules provides evidence of a length-dependent persistent length, PNAS, 103 (27), 10248-10253, (2006), https://dx.doi.org/10.1073/pnas.0603931103.

\section{APPENDIX A}

$$
\begin{array}{r}
A_{1}=-C_{11} h ; \\
A_{2}=\frac{C_{66} l_{z}^{2} h}{4 R^{2}} ; \\
A_{3}=-\frac{h}{R^{2}}\left(\frac{C_{55} l_{\theta}^{2}+C_{66} l_{z}^{2}}{2 R^{2}}+C_{66}\right) ; \\
A_{4}=\frac{C_{66} l_{z}^{2} h}{4 R^{4}} ; \\
A_{6}=\frac{C_{66} l_{z}^{2} h}{4 R} ; \\
\left.\frac{A_{55} l_{\theta}^{2}}{2 R^{2}}-\frac{\left(C_{44} l_{x}^{2}+C_{66} l_{z}^{2}\right)}{8 R^{2}}-C_{12}-C_{66}\right) ; \\
A_{7}=-\frac{C_{66} l_{z}^{2} h}{4 R^{3}} ; \\
A_{8}=-\frac{C_{12} h}{R} ; \\
\left.A_{9}=-\frac{C_{44} l_{x}^{2}-C_{66} l_{z}^{2}}{4 R^{3}}\right) ; \\
A_{10}=\frac{C_{55} l_{2}^{2}-\frac{h_{11}}{8 R^{2}}\left(C_{44} l_{x}^{2}-3 C_{66} l_{z}^{2}\right)}{h}\left(C_{55} l_{\theta}^{2}+\frac{3 C_{66} l_{z}^{2}}{2}\right) ;
\end{array}
$$

$$
\begin{array}{r}
B_{1}=\frac{C_{66} l_{z}^{2} h}{4} ; \\
B_{2}=h\left(\frac{C_{66} l_{z}^{2}}{4 R^{2}}-\frac{C_{44} l_{x}^{2}}{4 R^{2}}-\frac{C_{55} l_{\theta}^{2}}{2 R^{2}}-C_{66}\right) ; \\
B_{3}=-\frac{h}{R^{2}}\left(C_{22}+\frac{C_{44} l_{x}^{2}}{4 R^{2}}\right) ; \\
B_{4}=\frac{C_{66} l_{z}^{2} h}{4 R^{2}} ; \\
B_{5}=\frac{h}{R^{2}}\left(\frac{C_{44} l_{x}^{2}}{4 R^{2}}+k_{s} C_{44}\right) ; \\
B_{6}=-\frac{C_{66} l_{z}^{2} h}{4 R} ; \\
B_{7}=-\frac{C_{66} l_{z}^{2} h}{4 R^{3}} ; \\
\frac{h}{R}\left(\frac{C_{55} l_{\theta}^{2}}{2 R^{2}}-\frac{C_{44} l_{x}^{2}+C_{66} l_{z}^{2}}{8 R^{2}}-C_{12}-C_{66}\right) ;
\end{array}
$$$$
B_{9}=\frac{h}{4 R^{2}}\left(C_{44} l_{x}^{2}+\frac{3 C_{55} l_{\theta}^{2}}{2}+\frac{C_{66} l_{z}^{2}}{2}\right) ;
$$$$
B_{10}=\frac{C_{44} l_{x}^{2} h}{4 R^{4}} ;
$$$$
B_{11}=-\frac{h}{R^{2}}\left(C_{22}+\frac{C_{44} l_{x}^{2}}{4 R^{2}}+k_{s} C_{44}\right) \text {; }
$$$$
B_{12}=-\frac{C_{44} l_{x}^{2} h}{4 R^{3}} ;
$$$$
B_{13}=-\frac{h}{4 R^{2}}\left(\frac{3 C_{55} l_{\theta}^{2}}{2}-\frac{C_{44} l_{x}^{2}}{2}+C_{66} l_{z}^{2}\right) \text {; }
$$$$
B_{14}=-\frac{h}{8 R}\left(5 C_{44} l_{x}^{2}+C_{66} l_{z}^{2}\right) ;
$$$$
B_{15}=-\frac{h}{R}\left(\frac{C_{44} l_{x}^{2}}{4 R^{2}}+k_{s} C_{44}\right) \text {; }
$$

$$
\begin{array}{r}
F_{1}=\frac{C_{55} l_{\theta}^{2} h}{4} ; \\
F_{2}=-h\left(\frac{C_{66} l_{z}^{2}}{4 R^{2}}+k_{s} C_{55}\right) ; \\
F_{3}=\frac{h}{4 R^{2}}\left(C_{44} l_{x}^{2}+C_{55} l_{\theta}^{2}\right) ; \\
F_{4}=\frac{C_{44} l_{x}^{2} h}{4 R^{2}} ; \\
F_{5}=-\frac{h}{R^{2}}\left(\frac{C_{44} l_{x}^{2}}{4 R^{2}}+k_{s} C_{44}\right) ; \\
F_{7}=\frac{C_{12} h}{R} ; \\
F_{8}^{2}=-\frac{C_{44} l_{x}^{2} h}{4 R^{4}} ; \\
F_{11}=-\frac{C_{44} l_{x}^{2} h}{4 R^{3}} ; \\
F_{10}=\frac{F_{9}}{2 R^{3}}\left(C_{45} l_{\theta}^{2}-\frac{C_{44} l_{x}^{2}}{4}+\frac{C_{66} l_{z}^{2}}{4}\right) ; \\
C^{2}\left(C_{55} l_{\theta}^{2}\right. \\
2
\end{array}
$$




$$
\begin{aligned}
& F_{13}=-\frac{C_{55} l_{\theta}^{2}}{4} ; \\
& F_{14}=h\left(\frac{C_{66} l_{z}^{2}}{4 R^{2}}-k_{s} C_{55}\right) ; \\
& F_{15}=-\frac{h}{8 R^{2}}\left(3 C_{55} l_{\theta}^{2}-C_{44} l_{x}^{2}\right) \text {; } \\
& F_{16}=-\frac{h}{R}\left(\frac{C_{44} l_{x}^{2}}{4 R^{2}}+k_{s} C_{44}\right) ; \\
& F_{17}=-\frac{h}{8 R}\left(3 C_{44} l_{x}^{2}-C_{55} l_{\theta}^{2}\right) \text {; } \\
& D_{1}=-\frac{h}{4}\left(\frac{C_{11} h^{2}}{3}+C_{55} l_{\theta}^{2}\right) ; \\
& D_{2}=\frac{C_{66} l_{z}^{2} h^{3}}{48 R^{2}} ; \\
& D_{3}=\frac{C_{66} l_{z}^{2} h^{3}}{48 R^{4}} ; \\
& D_{4}=\frac{C_{55} l_{\theta}^{2} h}{4} ; \\
& D_{5}=-\frac{h}{2 R^{2}}\left(C_{55} l_{\theta}^{2}+C_{66} l_{z}^{2}+\frac{C_{66} h^{2}}{6}\right) ; \\
& E_{7}=-\frac{1}{R}\left(\frac{C_{12} h^{3}}{12}+\frac{C_{66} h^{3}}{12}-\frac{C_{66} l_{z}^{2} h}{2}+\right. \\
& \left.-\frac{C_{44} l_{x}^{2} h+C_{55} l_{\theta}^{2} h}{8}\right) ; \\
& E_{8}=-\frac{1}{R}\left(\frac{3 C_{66} l_{z}^{2} h}{8 R}-\frac{C_{44} l_{x}^{2} h}{8 R}\right) ; \\
& E_{9}=-\frac{1}{R}\left(\frac{C_{44} l_{x}^{2} h}{4 R^{2}}+k_{s} C_{44} h\right) ; \\
& E_{10}=\frac{C_{44} l_{x}^{2} h}{4 R^{3}} ; \\
& E_{11}=-\left(\frac{5 C_{44} l_{x}^{2} h}{8 R}+\frac{C_{66} l_{z}^{2} h}{8 R}\right) ; \\
& E_{12}=\frac{1}{R}\left(\frac{3 C_{44} l_{x}^{2} h}{8}-\frac{C_{55} l_{\theta}^{2} h}{8}\right) ; \\
& E_{13}=\frac{1}{R}\left(\frac{C_{44} l_{x}^{2} h}{4 R^{2}}+k_{s} C_{44} h\right) ; \\
& E_{14}=-\frac{C_{44} l_{x}^{2} h}{4 R^{3}} ; \\
& E_{15}=\left(k_{s} C_{44} h+\frac{C_{14} l_{x}^{2} h}{4 R^{2}}\right) ;
\end{aligned}
$$$$
D_{6}=h\left(\frac{C_{66} l_{z}^{2}}{4 R^{2}}+k_{s} C_{55}\right) ;
$$$$
D_{7}=-\frac{h}{2 R}\left(\frac{C_{12} h^{2}}{6}+\frac{C_{66} h^{2}}{6}-C_{66} l_{z}^{2}+\right.
$$$$
\left.-\frac{C_{55} l_{\theta}^{2}+C_{44} l_{x}^{2}}{4}\right)
$$$$
D_{8}=-\frac{C_{66} l_{z}^{2} h^{3}}{48 R^{3}}
$$$$
D_{9}=-\frac{C_{66} l_{z}^{2} h^{3}}{48 R} ;
$$$$
D_{10}=\frac{h}{2 R^{3}}\left(C_{55} l_{\theta}^{2}+\frac{3 C_{66} l_{z}^{2}}{2}\right) ;
$$$$
D_{11}=-h\left(\frac{C_{66} l_{z}^{2}}{4 R^{2}}-k_{s} C_{55}\right) ;
$$$$
D_{12}=\frac{h}{8 R^{2}}\left(3 C_{55} l_{\theta}^{2}-C_{44} l_{x}^{2}\right) ;
$$$$
D_{13}=-\frac{h}{4 R^{2}}\left(\frac{3 C_{55} l_{\theta}^{2}}{2}-\frac{C_{44} l_{x}^{2}}{2}+C_{66} l_{z}^{2}\right) \text {; }
$$

\section{APPENDIX B}

$$
\begin{array}{r}
a_{1}=C_{11} \varepsilon_{x x}+C_{12} \varepsilon_{\theta \theta} ; \\
a_{2}=C_{21} \varepsilon_{x x}+C_{22} \varepsilon_{\theta \theta} ; \\
a_{3}=C_{44} \varepsilon_{\theta z} ; \\
a_{4}=C_{55} \varepsilon_{x z} ; \\
a_{5}=C_{66} \varepsilon_{\theta x} ; \\
b_{1}=2 l_{x}^{2} G_{x x} \chi_{x x} ; \\
b_{2}=2 l_{\theta}^{2} G_{\theta \theta} \chi_{\theta \theta} ; \\
b_{3}=2 l_{z}^{2} G_{z z} \chi_{z z} ; \\
b_{4}=l_{x}^{2} G_{x \theta} \chi_{x \theta}+\left[\frac{l_{\theta}^{2} G_{\theta x}+l_{x}^{2} G_{x \theta}}{2}\right] \chi_{\theta x} ; \\
b_{5}=l_{\theta}^{2} G_{\theta x} \chi_{\theta x}+\left[\frac{l_{\theta}^{2} G_{\theta x}+l_{x}^{2} G_{x \theta}}{2}\right] \chi_{x \theta} ; \\
b_{6}=l_{x}^{2} G_{x z} \chi_{x z}+\left[\frac{l_{z}^{2} G_{z x}+l_{x}^{2} G_{x z}}{2}\right] \chi_{z x} ; \\
b_{7}=l_{z}^{2} G_{z x} \chi_{z x}+\left[\frac{l_{z}^{2} G_{z x}+l_{x}^{2} G_{x z}}{2}\right] \chi_{x z} ; \\
b_{9}=l_{z}^{2} G_{z \theta} \chi_{z \theta}+\left[\frac{l_{\theta}^{2} G_{\theta z}+l^{2} z G_{z \theta}}{2}\right] \chi_{\theta z} ;
\end{array}
$$$$
E_{1}=\frac{C_{66} l_{z}^{2} h^{3}}{48} ;
$$

$$
\begin{array}{r}
E_{2}=-\left(\frac{C_{66} h^{3}}{12}+\frac{C_{44} l_{x}^{2} h}{2}+\frac{C_{55} l_{\theta}^{2} h^{3}}{24 R^{2}}+\frac{C_{66} l_{z}^{2} h}{2}\right) ; \\
E_{3}=\frac{C_{66} l_{z}^{2} h^{3}}{48 R^{2}} ;
\end{array}
$$$$
E_{4}=-\frac{1}{R^{2}}\left(\frac{C_{22} h^{3}}{12}+\frac{C_{44} l_{x}^{2} h}{4}\right) ;
$$$$
E_{5}=-\frac{C_{66} l_{z}^{2} h^{3}}{48 R^{3}}
$$$$
E_{6}=-\frac{C_{66} l_{z}^{2} h^{3}}{48 R} ;
$$ 